\title{
Las consecuencias sobre los derechos de las mujeres por la información que da la televisión de Ayahualulco
}

\author{
Karen Flores Arteaga ${ }^{1}$ \\ Alejandra García Ortiz ${ }^{2}$ \\ Alma Sarai Monsalvo Medina ${ }^{3}$ \\ Karla María Zúñiga Santillán ${ }^{4}$ \\ Asesora: Dra. Esther Fragoso Fernández
}

\section{Resumen}

La violencia que sufren las mujeres en nuestro país es fríamente naturalizada o vista como una rutina diaria, muchas de las afectadas no saben distinguir entre el buen trato y el mal trato, no saben qué hacer o con quién acudir para terminar con los golpes, insultos y todas las acciones que violen sus derechos; es por esto que la investigación se enfoca al estudio de las consecuencias que trae a la vida de las mujeres la información transmitida en la televisión con relación a sus derechos individuales y a identificar el grado de conocimiento sobre la violencia que tienen las mujeres de la comunidad Ayahualulco, en el estado de Veracruz.

\section{Introducción}

\section{Problema de investigación}

En México se comete abuso contra la integridad de las mujeres. Anualmente 8,656,871 mujeres son maltratadas, esto sucede específicamente en las zonas rurales. Así que decidimos investigar de qué manera la televisión como medio de comunicación incide en la información sobre los derechos humanos de las mujeres y de la misma manera ayudar a combatir los abusos contra la mujer.

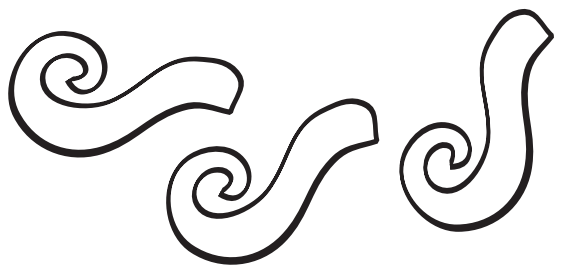

Objetivos

General

- Identificar los abusos que se comenten en contra de la mujer y analizar el tipo de información que la televisión puede proporcionar a favor de los derechos humanos de las mujeres en la comunidad de Ayahualulco.

\section{Específicos}

- Reconocer los aspectos de la programación de la televisión abierta en México, relacionados con los derechos humanos de la mujer.

- Clasificar aspectos promotores de los derechos humanos de la mujer y aquellos que los destruyen.

- Identificar los contenidos de los programas de la televisión y su relación con la vida cotidiana de las mujeres de Ayahualulco.

- Encontrar alternativas viables para la promoción y respeto de los derechos humanos de la mujer de aquella comunidad.

1 Sétimo semestre de la Licenciatura en Ciencias de la Educación.

2 Sétimo semestre de la Licenciatura en Ciencias de la Educación. (ago_2426@hotmail.com)

3 Sétimo semestre de la Licenciatura en Ciencias de la Educación. (almapastrana@hotmail.com)

4 Sétimo semestre de la Licenciatura en Ciencias de la Educación. (karla_m_zs@hotmail.com) 


\section{Preguntas de Investigación}

1. ¿Qué tipo de abusos contra los derechos de las mujeres se comenten en Ayahualulco?

2. ¿Cuáles son las causas que generan estos abusos?

3. ¿Cuáles son los medios de comunicación más influyentes en aquella comunidad?

4. ¿Qué características tiene la información referente a los derechos humanos de la mujer que se transmite en los medios masivos de comunicación en Ayahualulco?

5. Desde la perspectiva de la comunicación ¿Cómo pueden ser combatidos los abusos y ser identificadas las necesidades de la mujer?

\section{Justificación}

PERLA es el Proyecto Educativo Regional Lasallista Latinoamericano en el que se exponen los principales temas y puntos a tratar para mejorar la calidad educativa; la urgencia que queremos abordar dentro de nuestra investigación de acuerdo con el proyecto PERLA, es la 1.5 “Una educación en y desde el respeto a los Derechos Humanos". Esta urgencia tiene como necesidad impulsar proyectos en pro de la defensa de los derechos humanos y el medio para lograr el fortalecimiento de las familias, siempre enfocado a situación de injusticia, violencia, abuso y explotación, en el caso de nuestra investigación, específicamente en las mujeres.

Parte importante de esto es lograr crear una conciencia y conocimiento sobre las problemáticas antes mencionadas y los organismos defensores de los derechos humanos, así como un buen manejo de información y programas de acción para frenar esa situación de violencia hacia las mujeres en esta comunidad, mediante obras lasallistas.

\section{Marco histórico}

Desde el inicio de las civilizaciones ha existido una separación entre los géneros. La mujer ha sido catalogada como más débil y su rol casi siempre ha sido el de ama de casa, esto ha influido mucho en la vida de las personas y ha dado la pauta para que el hombre se sienta con poder sobre la mujer y la abuse, atentando con sus derechos e igualdad. (indujeres2012)

Se han creado leyes, programas y cursos para disminuir la violencia hacia las mujeres, el primero es el principio fundamental de igualdad jurídica entre hombres y mujeres se integró a la Constitución Política de los Estados Unidos Mexicanos en 1974, mediante la reforma al Artículo 4º. (INEGI 2010).

En 1980 se creó el Programa Nacional de Integración de la Mujer al Desarrollo que promueve el mejoramiento de la condición social de las mujeres. (INEGI 2010).

Declaración de Naciones Unidas sobre la Eliminación de la Violencia contra la Mujer 1993. (INEGI 2010).

De 1993 hasta enero de 2006, en Ciudad Juárez Chihuahua más de 430 mujeres han sido asesinadas y más de 600 están desaparecidas. (El Universal).

Los primeros esfuerzos que al respecto se hicieron para contar con estadísticas son: la Encuesta sobre Organización Doméstica, levantada en 1994 por el Centro de Investigación y Estudios Superiores en Antropología Social (CIESAS) y la Encuesta sobre Organización Familiar, aplicada también en ese mismo año por el Grupo de Educación Popular con Mujeres (GEM, AC). Posteriormente, en 1998, el Colegio de México aplica la Encuesta sobre Dinámica Familiar y un año después, el INEGI realiza la Encuesta sobre Violencia Intrafami- 


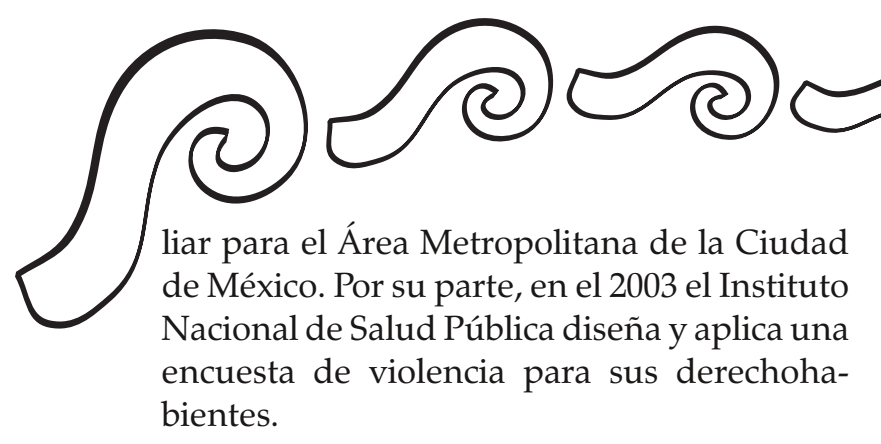

En 2003 se reporta que un total de 19, 471, 972 de mujeres de 15 años y más con pareja residente en el hogar, son violentadas 9, 064, 458, lo que señala una proporción de violencia de casi la mitad del universo estudiado: una de cada dos mujeres.

De 2004 a 2009 los casos aumentaron 12\% y 67\% de las mujeres mayores de 15 años en el país han sufrido algún tipo de maltrato. (INEGI 2010). Según el censo del 2006 hay 103, 263,388 mujeres en México de las cuales 8,656,871 son violentadas. En 2010 de 57, 481,307 mujeres, 9,683,779 mujeres son violentadas lo que muestra un aumento en la agresión hacia las mujeres ya sea emocional, física o verbal incluso se toma en cuenta la violencia laboral. (INEGI 2010).

Actualmente las mujeres siguen en su lucha por tener los mismos derechos, ya han logrado la igualdad ante la ley pero debido a la falta de información hace que algunas mujeres aún estén sometidas.

\section{Marco conceptual}

Violencia: "En términos generales podemos decir que la violencia es todo acto de abuso o coerción que una persona ejerce sobre otra y que puede darse en cualquier ámbito de las personas, es decir, en el trabajo, calle, hogar, escuela, etc." (Derecho a las mujeres a una vida libre de violencia en el ámbito familiar, pp.2).

Para esta investigación definiremos a la violencia como todo acto de abuso ejercido de una persona a otra en cualquier ámbito.

Violencia contra las mujeres: "Es toda acción u omisión que, basada en su género y deriva- da del uso y/o abuso del poder, tenga por objeto o resultado un daño o sufrimiento físico, psicológico, patrimonial, económico, sexual o la muerte en las mujeres, tanto en el ámbito público como privado, que limite su acceso a una vida libre de violencia". (Derecho a las mujeres a una vida libre de violencia en el ámbito familiar, pp.2).

El concepto de violencia contra las mujeres lo definiremos como la acción u omisión que tenga por objeto o resulte un daño en la mujer que no le permita vivir una vida libre de violencia.

\section{Tipos de violencia}

Violencia psicoemocional: "Toda acción u omisión dirigida a desvalorar, intimidar o controlar sus acciones, comportamientos y decisiones, consistente en prohibiciones, condicionamientos, intimidaciones, insultos, celotipia, descuido, chantaje, humillaciones, abandono, o cualquier otra que provoque en quien lo recibe alteración autocognitiva y autovalorativa que integran su autoestima o alteraciones en alguna esfera o área de su estructura psíquica" (reglamento y ley de acceso de las mujeres a una vida libre de violencia, pp.9).

Para esta investigación definiremos violencia psicoemocional como toda acción u omisión hacia una persona que daña su integridad y procesos psicológicos.

Violencia física: "Toda acción u omisión intencional que causa un daño en la integridad física". (Reglamento y ley de acceso de las mujeres a una vida libre de violencia, pp.10).

Para fines de la investigación utilizaremos la definición como acto que daña la integridad física de la persona.

Violencia patrimonial: “Toda acción u omisión que ocasiona daño o menoscabo en los bienes muebles o inmuebles de la mujer y su patri- 


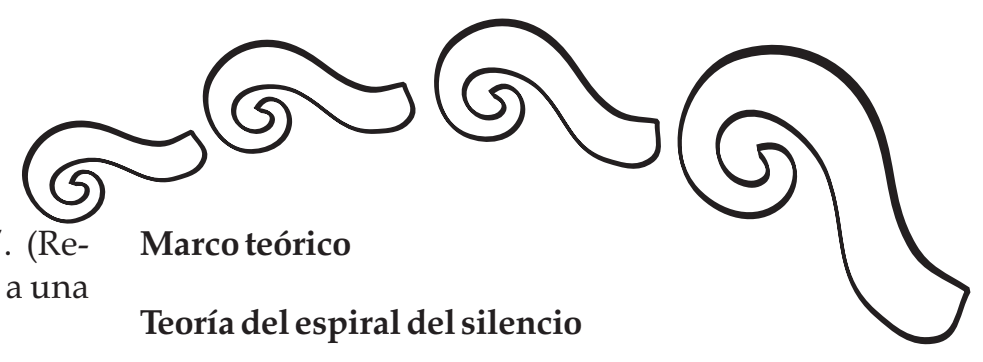

En el estudio la definiremos como la acción que ocasiona daño en los bienes, muebles o recursos económicos.

Violencia económica: “toda acción u omisión que afecta la economía de la mujer a través de las limitaciones, restricciones, negociaciones, de las percepciones económicas". (Reglamento y ley de acceso de las mujeres a una vida libre de violencia, pp.10).

La definiremos como la acción que perjudica la economía de la mujer.

Violencia Sexual: "toda acción u omisión que amenaza, pone en riesgo o lesiona la libertad, seguridad, integridad y desarrollo psicosexual de la mujer". (Reglamento y ley de acceso de las mujeres a una vida libre de violencia, pp.10).

Para esta investigación, nos referiremos a la violencia sexual como el acto que daña la integridad y desarrollo psicosexual de la mujer.

Derechos Humanos: "los derechos humanos son derechos inherentes a todos los seres humanos, sin distinción alguna de nacionalidad, lugar de residencia, sexo, origen nacional o étnico, color, religión, lengua o cualquier otra condición. Todos tenemos los mismos derechos, sin discriminación alguna. Estos derechos son interrelacionados, interdependientes e indivisibles". (http://www.ohchr.org/SP/ Issues/Pages/WhatareHumanRigths.aspx).

Definiremos a los derechos como aquella facultad humana para exigir el uso de la justicia con base en lo establecido por la ley.

Usaremos el concepto Humano, como aquella actividad, característica o situación particular del ser humano.

Elizabeth Noelle-Neumann crea la teoría del espiral del silencio que nos dice que los medios de comunicación crean la opinión pública, que es la presión que se ejerce en el público y se genera el efecto cuando las opiniones conforman el clima general que sobre algún tema en cuestión pueda darse en una sociedad .Todo esto no es más que la opinión personal (valores, creencias y percepciones)

- La sociedad amenaza a los individuos desviados con el aislamiento.

- Los individuos experimentan un continuo miedo al aislamiento.

- Este miedo hace que los individuos evalúen el clima de la opinión.

- Esta evaluación influye en el comportamiento del público.

Con esta relación de estos 4 elementos, se llega a la hipótesis que los individuos tiende a callar frente al temor del aislamiento y que también los individuos se mantienen alertas acerca de las interpretaciones que fluyen en su entorno, cuáles son las correctas y cuáles no. Y el efecto de la teoría del silencio es más, cuando son temas significativos para el individuo.

Los discursos sociales, que revelan una actitud frente a algún hecho, posibilitan la transformación de una opinión en una conducta observable, y que a la larga puede generar un cambio de conducta. El efecto de amplificación de la realidad producido por los medios, permite reforzar el efecto de la espiral del silencio. Los medios suministran a la gente las palabras y las frases que pueden utilizar para defender un punto de vista, si la gente no encuentra expresiones habituales repetidas con frecuencia, a favor de su punto de vista, cae en el silencio o se vuelve muda.( Portillo Sánchez Maricela/Esterna Madrid Javier, 1998) 


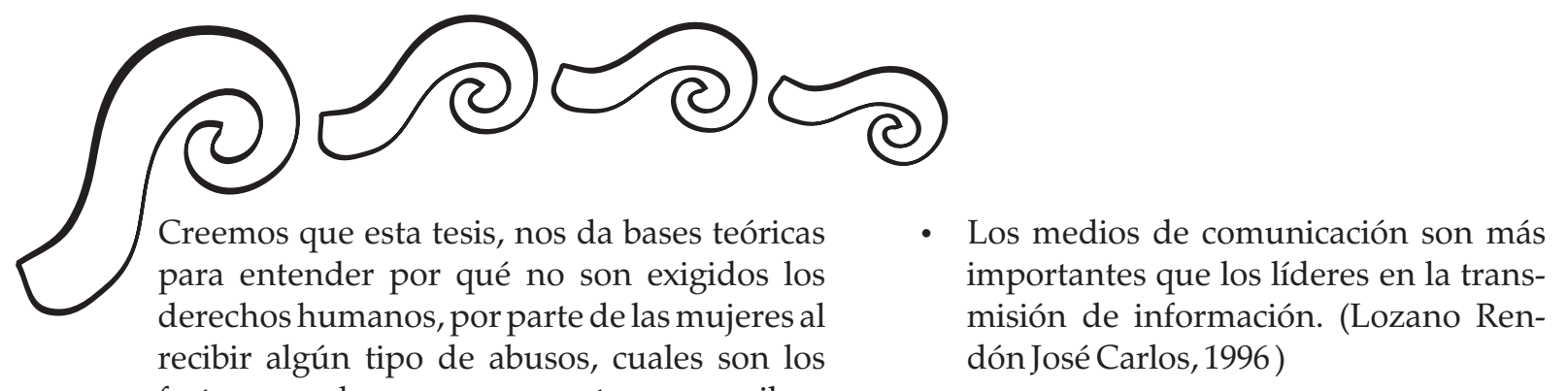
factores que hacen que se mantengan en silencio y que muchas veces sean vistos de forma natural.

\section{Influencia personal}

En la influencia personal, destaca la importancia de los contactos personales sobre la exposición a los medios masivos de comunicación, o del flujo de la comunicación en dos pasos, que enfatiza el proceso de circulación de información y opinión de los medios a los líderes y de estos a sus seguidores. Los medios, más que cambiar la actitud de los receptores hacia ciertas personas, refuerzan las predisposiciones.

En el proceso de comunicación de dos pasos, existen varias tendencias:

- Personas educadas acuden más a los medios.

- Selección de las opiniones con las que están de acuerdo.

- Las opiniones reciben mayor atención de aquellos que están interesados y ya tienen una opinión formada.

- Aquellos que están más informados, están menos dispuestos al cambio.

- Se ven afectados más por decisiones por el contacto personal, que por los medios masivos.

- El líder puede ser de cualquier clase social, pero está un poco más informado que la masa.

- Sólo se es líder en algunos campos.

- Los líderes de opinión son accesibles, hacen competencia, saben del tema, conocen a muchas personas.

\section{La divulgación de las nuevas ideas y prácticas}

Los cambios sociales eran una característica sólo de la sociedad americana, mismos que ahora ya también son del mundo por medio de procesos, la divulgación; uno de estos procesos se puede entender como una nueva idea o hábito que se da en cierto tiempo, con una estructura social y canales de emisión específicos. Durante los años treinta hubo un desarrollo de estudios que investigaban las innovaciones sociales y comenzaron a estudiar la difusión social y con la creación de la radio se comenzó con la comunicación colectiva.

El interés por la difusión actualmente es por parte de los sociólogos rurales y en otro lado personas con intereses comunes en comunicación de masas, los investigadores intentan cambiar formas de pensar, actuar y hablar mediante un modelo que se compone de:

- Medios omnipotentes capaces de dar ideas a mentes desamparadas.

- Un público de masas atomizados, relacionado con los medios de masas.

Aunque se menciona que por medio de estos modelos es difícil cambiar la forma de pensar de una persona, por medio de los medios de comunicación, ya que estos medios no tienen tanto poder de convencimiento y más bien la influencia en el cambio de opinión la tenían las personas, creando el "flujo de comunicación de dos pasos", el cual dice que la influencia va de los medios a los líderes de opinión y estos influyen en su grupo social, se puede observar que:

- La influencia de otras personas sobre decisiones específicas puede ser mejor.

- Los influyentes tienen relación estrecha con quien los influye. 


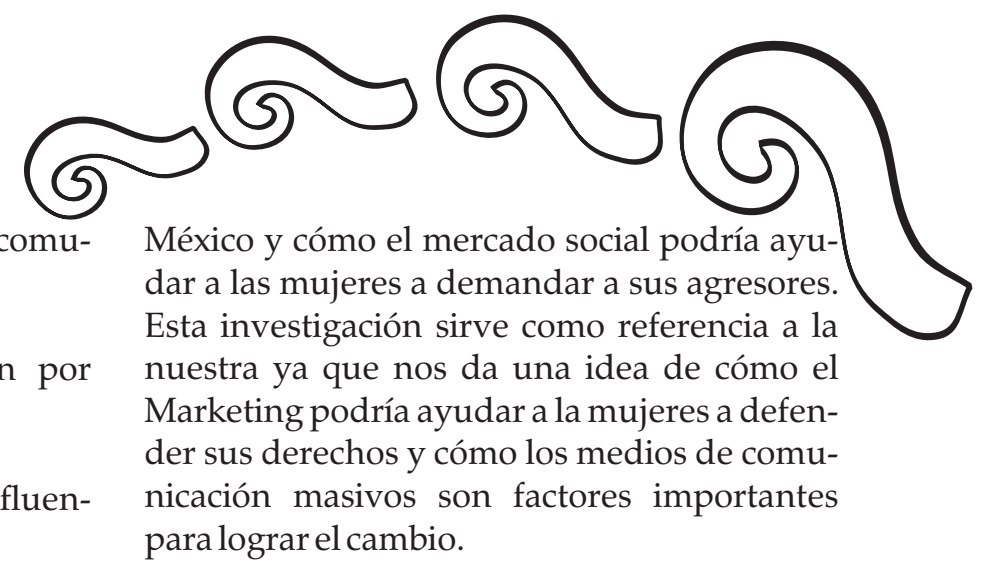

- Los líderes de opinión estas en contacto con los medios masivos.

Una de las razones por la que existe una barrera entre investigadores de la comunicación y sociólogos rurales es el producto de las imágenes opuestas de la sociedad que existen en la mente de cada individuo.

Los sociólogos se han centrado en etapas de la toma de decisiones dentro del individuo

- Etapa inicial: algunos adoptantes prueban la innovación, confiando en la información que les dan.

- Etapa intermedia: innovación fue rápida, por la influencia que ejercen.

- Etapa final: hasta los más apegados ceden.(Katz/compilación de Schramm , 1982)

Esta teoría nos ayudará a comprender cómo los medios de comunicación en el caso específico de nuestra investigación, como la televisión y los líderes de opinión que aquí se desenvuelven, actúan como mediadores de la información. Veremos si la divulgación de los derechos humanos sobre mujeres abusadas, si es emitido por este medio y si las mujeres de esta comunidad realmente cambian su forma de pensar o están más informadas de esta situación gracias a los medios de comunicación.

\section{Marco referencial}

\section{Marketing Social}

Alumnos de la Universidad de las Américas Puebla (www.udlap. $m x$ ) realizaron una investigación acerca del maltrato a las mujeres en

"El marketing social, como señala Acosta (1985) busca estudiar las interacciones sociales e incorporar la idea de aplicar conceptos del marketing para detectar y diagnosticar los patrones que faciliten el análisis de oportunidades al solucionar los conflictos sociales, por lo que es una de las mejores herramientas que pueden ayudar a solucionar el problema del maltrato a la mujer. Así el proceso del marketing social permite un acercamiento directo con las víctimas del maltrato para recolectar datos y del análisis de estos se establecen los bienes satisfactorios que cubran los deseos y aspiraciones de los clientes".

Durante el desarrollo de este proyecto de investigación se conocerán y aplicarán los elementos que conforman el marketing social para la elaboración de un programa de comunicación social que se enfocará al maltrato de la mujer, a fin de encontrar un producto social que permita mejorar la calidad de vida de las mujeres maltratadas.

El objetivo general de la investigación citada anteriormente es interesante, pero a gran escala, lo que genera un problema para llevarlo a cabo, aunque no imposible.

En cuanto a resultados mencionan lo siguiente:

"Las mismas mujeres sugieren que la solución está en manos de las madres quienes deben enseñar a sus hijos que existe una igualdad de género y las mujeres y hombres tienen el mismo valor como seres humanos. En este caso, son las mujeres quienes deben recurrir a cursos que les ayuden a cambiar los patrones que degeneran la dignidad de las mujeres y les 


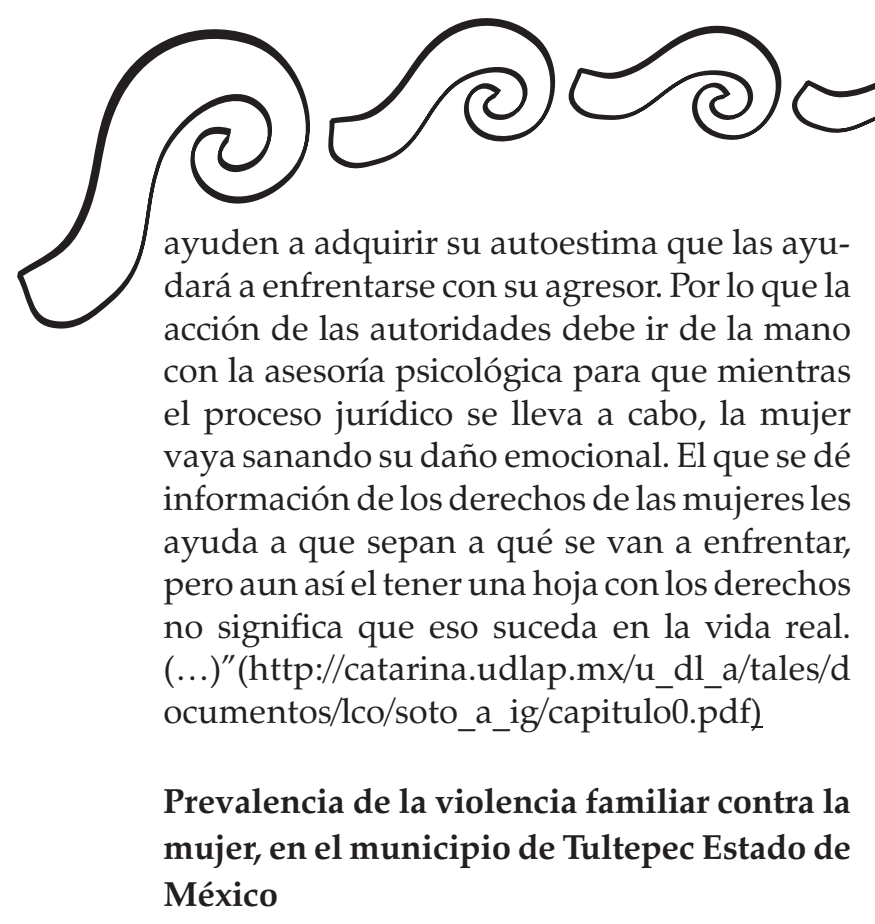

La sexóloga Blanca Aidé Solano López hizo una investigación titulada Prevalencia de la violencia familiar contra la mujer, en el municipio de Tultepec Estado de México la cual a realizó a 521 mujeres entre 20 y 59 años, por medio de encuestas en 19 barrios y colonias de Tultepec, estado de México.

Los resultados que lanzó su investigación fueron que el $64 \%$ de las mujeres encuestadas no conocen sus derechos como mujer y el $78 \%$ no conoce alguna ley que le ayude en caso de ser maltratada. Como conclusión mencionó que hace falta educación en materia de derechos de la mujer y leyes que ayuden a las mujeres que viven violencia familiar. (http://www.slideshare.net/ozuani/violenciacontra-la-mujer-presentation)

Como podemos notar, en la zona de Tultepec Estado de México, las mujeres conocen información que las puedan ayudar, lo cual apoya nuestra investigación porque así sabremos si las mujeres en esa zona se encuentran en las mismas situaciones y podremos orientarlas un poco acerca de la violencia de distintos tipos.
Día internacional para la eliminación de la violencia contra la mujer

El INEGI se presenta una selección de datos socio demográficos para evidenciar algunas de las facetas de la violencia contra las mujeres, y al mismo tiempo informa que ha publicado el documento Las mujeres violentadas por su pareja en México, con base en los resultados de la Encuesta Nacional sobre la Dinámica de las Relaciones en los Hogares 2003, para enriquecer el conocimiento sobre el fenómeno de la violencia conyugal al analizar la gravedad y severidad, así como los efectos y consecuencias en la integridad física y emocional de las mujeres.

Algunos datos importantes son:

- De las mujeres de 15 años y más que viven con su pareja, $47 \%$ presenta algún tipo de violencia conyugal.

- De cada cien mujeres de 15 años y más que viven con su pareja, nueve señalan ser objeto de agresiones físicas y ocho de cada cien padecen violencia de tipo sexual.

- La violencia emocional sola o en combinación con otros tipos de violencia es la más frecuente entre las mujeres, $82.5 \%$ de las mujeres que padecen violencia por parte de su pareja declara insultos, amenazas, humillaciones e intimidaciones.

- Diez por ciento de las mujeres violentadas sufren violencia severa, la cual pone en riesgo su integridad física y emocional.

- Nueve de cada diez víctimas de delitos sexuales son mujeres.

- Diariamente mueren en promedio 


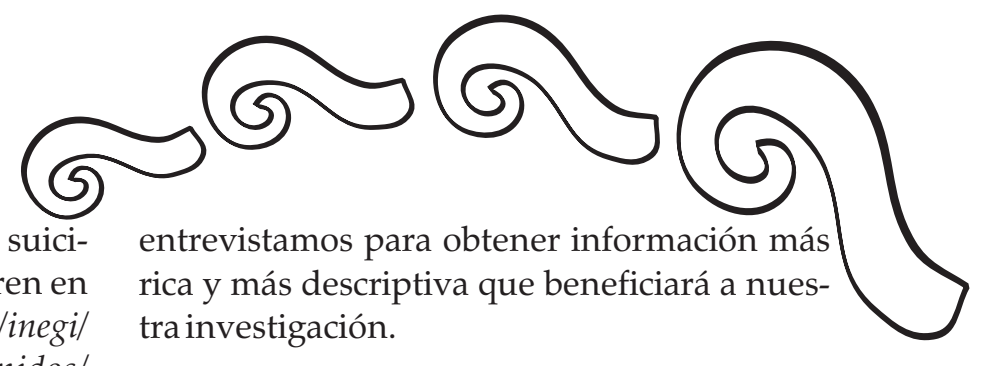

cinco mujeres por homicidios y suicidios y poco más de la mitad ocurren en la vivienda. (www.inegi.org.mx/inegi/ contenidos/espanol/prensa/contenidos/ estadisticas/2006/violencia06.pdf).

\section{Diseño}

Estos datos de investigación son útiles para nuestro trabajo porque muestran encuestas a nivel nacional acerca del maltrato a las mujeres, lo cual es importante ya que nosotros podremos observar a una sola entidad y tratar de hacer un cambio social. Quizá este tipo de soluciones puedan incrementar y así lograr un cambio más grande.

\section{Metodología}

\section{Enfoque}

En la investigación realizada, el enfoque que utilizamos es cualitativo (se caracteriza por la subjetividad, una perspectiva interna y va orientado hacia el proceso).

Las causas por las cuales se seleccionó este tipo de enfoque con: la creencia e importancia de conocer la opinión y percepción de cada uno de los alumnos con algunas preguntas abiertas, para saber qué es lo que predomina en la universidad de acuerdo con nuestro tema y tener una investigación más completa y mejor fundamentada, para poder obtener una resolución de la hipótesis con más validez.

\begin{abstract}
Alcance
El alcance de nuestra investigación es descriptivo, ya que con la investigación queremos llegar a un nivel de descripción amplio e identificar las características para desarrollarlas. Se tocarán varios detalles acerca de la vida de las mujeres entrevistadas y cómo es que la televisión ha influido en estos.
\end{abstract}

De igual forma ubicaremos variables que influyen en su comportamiento mientras las
Nuestra investigación es de tipo no experimental, porque no aplicamos ningún tipo de experimento, para recabar la información; estaremos en Ayahualulco una semana conviviendo con los sujetos de investigación y aplicando entrevistas y la observación para llegar a nuestro fin, constatamos a través de la aplicación individual de una entrevista semiestructurada sobre los derechos de las mujeres, el abuso que puede existir hacia ellas y la influencia que genera en este problema la televisión. Dentro de la no experimental, nuestra investigación es transeccional o transversal, porque sólo se refiere al conocimiento actual que se tiene sobre los abusos a las mujeres y sus derechos.

\section{Contexto}

La investigación se realizará en la comunidad de Ayahualulco, Veracruz. México. Donde hay un aproximado de 23, 743 mujeres que viven violentadas, ya sea física, psicológica o mentalmente. (INEGI, 2012)

Ayahualulco es una comunidad que tiene pocos servicios sociales y escasos programas de información para las mujeres, es por ello que surge el interés de realizar la investigación, para conocer el grado de conocimiento sobre la violencia de las mujeres del lugar.

Un apoyo importante es la escuela secundaria y bachillerato La Salle Ayahualulco, pues brinda educación a jóvenes de la comunidad.

\section{Sujetos, universo y muestra}

Mujeres que habitan la comunidad de Ayahualco y comunidades aledañas. 


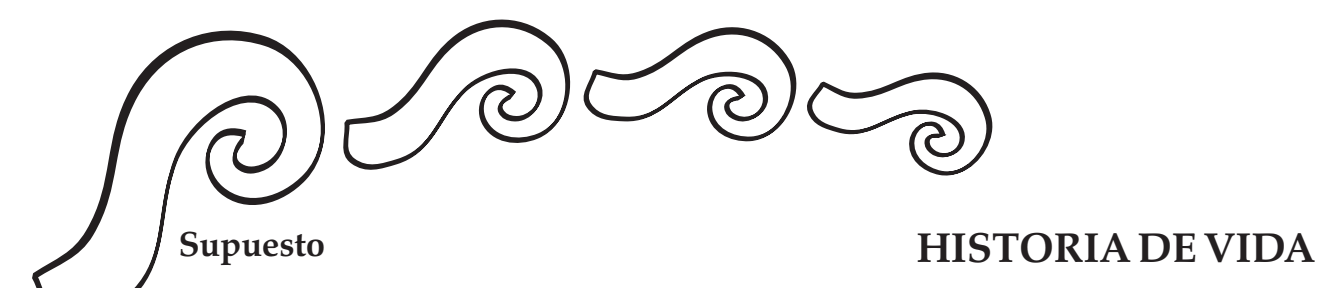

La televisión no informa ampliamente sobre las características del maltrato y las acciones que deben tomar las mujeres cuando sufren de violencia física o psicológica. La mayoría de las mujeres se informan por este medio de comunicación.

Nombre:

Edad:

Sexo:

\section{Procedimiento}

Visitar una semana el campo de investigación, Ayahualulco, Veracruz; en el cual comenzaremos nuestra investigación visitando varias casas en donde viven mujeres y aplicaremos entrevistas, las cuales nos ayudarán a comprobar nuestro supuesto. Trataremos de generar empatía para tener un mejor resultado en las respuestas esperando que sean honestas y amplias. Utilizaremos el método deductivo al momento de realizar las entrevistas para generar confianza en las personas y descubrir los sucesos que las mujeres han vivido.

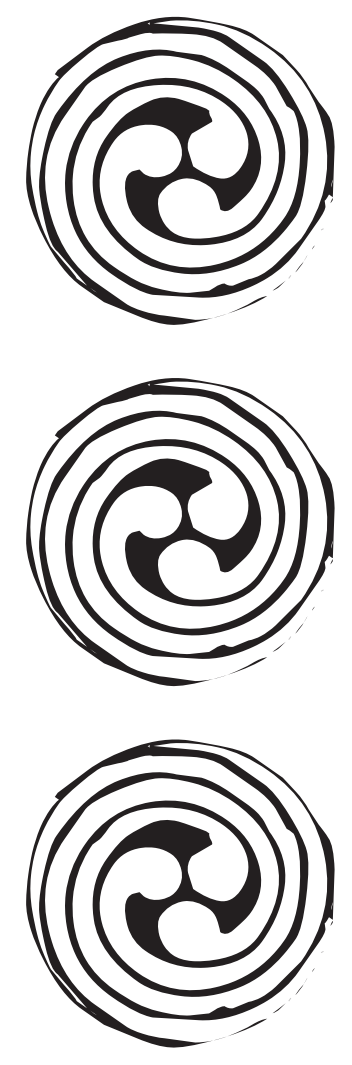

\section{Ocupación:}

\section{Indicaciones:}

- Narra el momento en que sentiste que tus derechos fueron violados, tu testimonio es valioso para la elaboración de instrumentos que nos permitan observar los cambios que ocurren en las mujeres que pasan por algo similar.

- Es importante que incluyas: ¿Cuándo sucedió? ¿Por qué sucedió? ¿Qué cambios hubo en tu persona? (físicos, psicológicos, económicos y sociales) ¿Cómo te sientes al respecto?

- Tu información es confidencial y exclusivamente el investigador tendrá acceso a ella.

- Puedes ocupar ambos lados de la hoja.

- Gracias por tu participación.

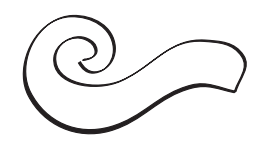




\section{INSTRUMENTOS}

FOLIO

\section{LAS CONSECUENCIAS SOBRE LOS DERECHOS DE LAS MUJERES POR LA INFORMACIÓN QUE DA LA TELEVISIÓN EN AYAHUALULCO}

NOMBRE:

EDAD: años

SEXO: M $\mathrm{F}$

OCUPACIÓN:

1. ¿Cuántos hijos tiene?
a) Ninguno
b) 1
c) 2
d) 3
e) 4 o más

2. ¿Tiene televisión en casa?
a) Sí
b) No

3. ¿Cuánto tiempo pasa su familia frente al televisor diariamente?
a) Nunca
b) Menos de
c) de 1 a 3
d) Más de 3 horas
una hora horas

4. ¿Qué tipo de programas ve?
a) Telenovelas
b) Series
c) Comedias
d) Noticias
e) Caricaturas
f) Todas las anteriores

5. ¿Sabe qué es violencia?
a) Sí
b) No
Defínala:

6. ¿Sabe cuáles son los tipos de violencia?
a) Sí
b) No

Menciónelos:

7. ¿Ha sufrido algún tipo de violencia?
a) Sí
b) No
¿Cuál?

8. ¿Cree usted que existe violencia familiar?
a) Sí
b) No

¿Cuál?

9. ¿Ha consultado la información televisiva para evitar este tipo de violencia?
a) Sí
b) No

10. ¿Esa información le ha ayudado a mejorar su situación?
a) Sí
b)No

¿Cómo? 


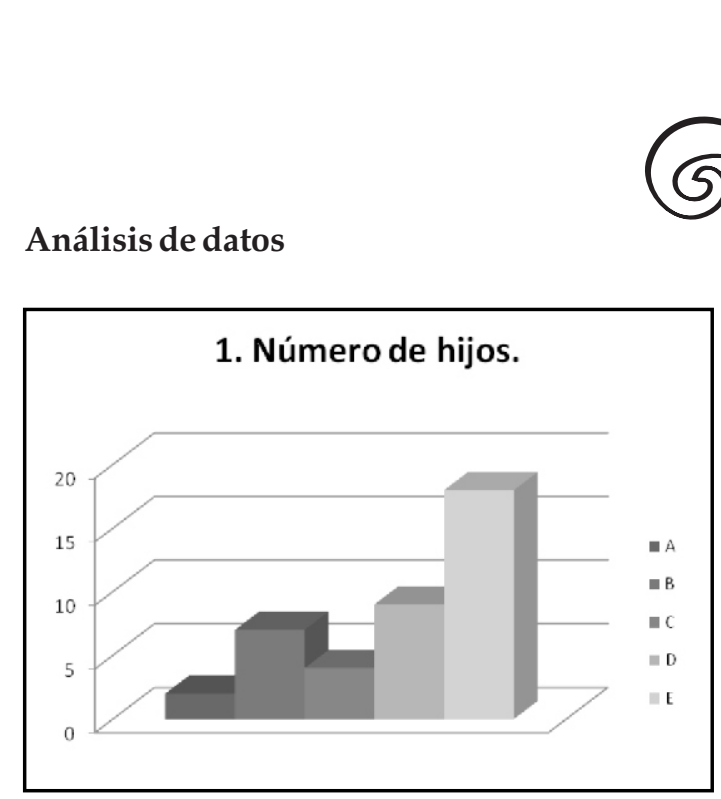
a) Ninguno
b) 1
c) 2
d) 3
e) 4 o más

Los resultados muestran que la mayoría de las mujeres en Ayahualulco tienen más de 4 hijos, después 3 hijos y el que obtuvo menos porcentaje fue la opción A, que significa que no tiene ningún hijo.

La teoría de La divulgación de las nuevas ideas y prácticas menciona que los medios deberían emitir información a mentes desamparadas, lo cual en este caso no se muestra que su objetivo haya sido alcanzado, porque la idea que las personas ahora tienen de una sociedad mejor es teniendo 1 o 2 hijos. En los resultados se ve que las personas de estas comunidades aún no tienen la idea de menos hijos para mejor vida en la mente.

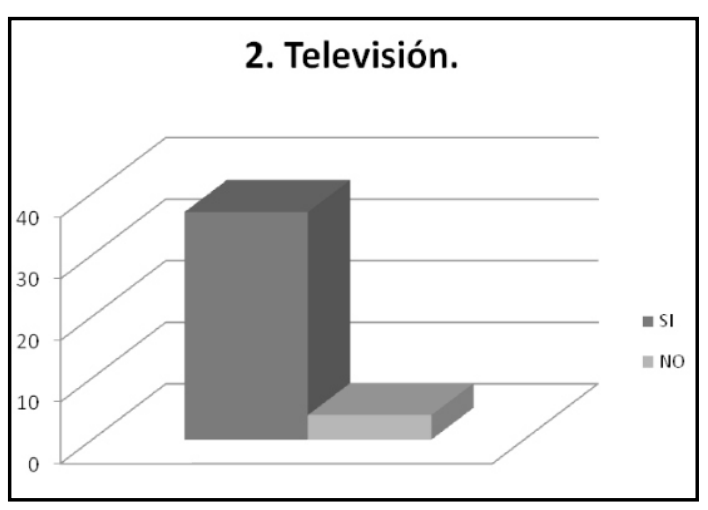

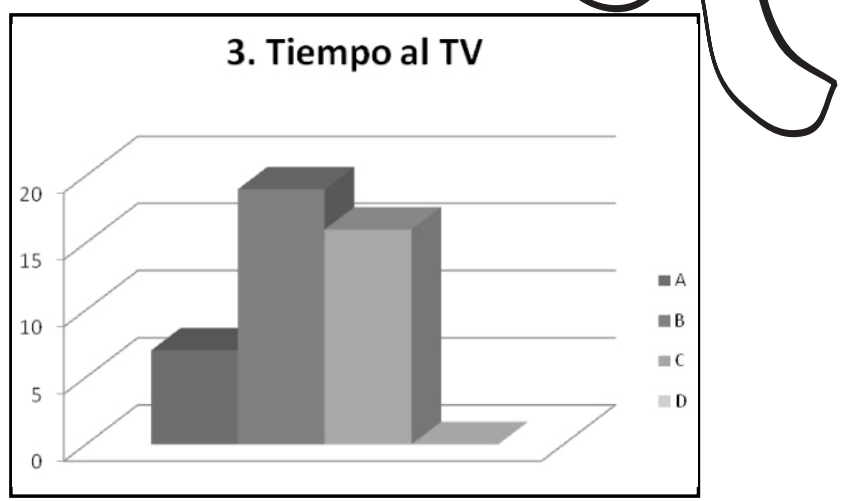
a) Nunca
b) Menos de una hora
c) De una a 3 horas
d) Más de 3 horas

Los resultados muestran que la mayoría de las mujeres en Ayahualulco tienen más de 4 hijos, después 3 hijos y el que obtuvo menos porcentaje fue la opción A, que significa que no tiene ningún hijo.

La teoría de La divulgación de las nuevas ideas y prácticas menciona que los medios deberían emitir información a mentes desamparadas, lo cual en este caso no se muestra que su objetivo haya sido alcanzado, porque la idea que las personas ahora tienen de una sociedad mejor es teniendo 1 o 2 hijos. En los resultados se ve que las personas de estas comunidades aún no tienen la idea de menos hijos para mejor vida en la mente.

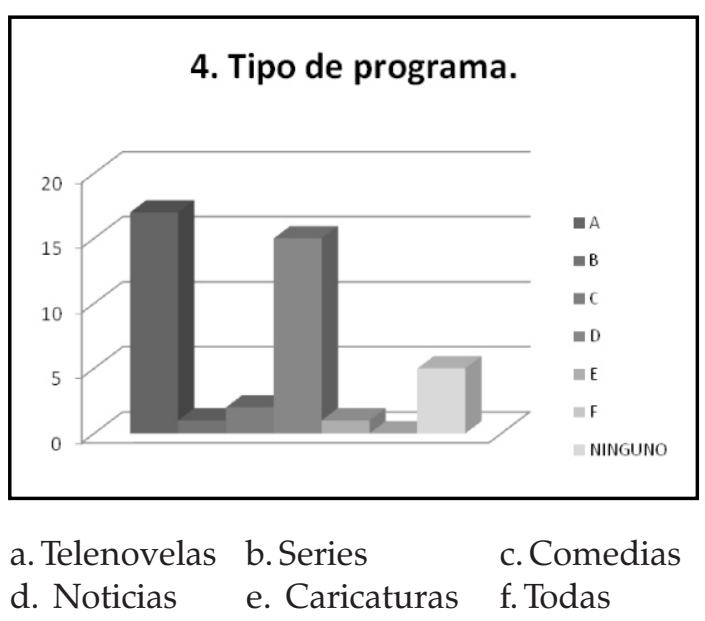



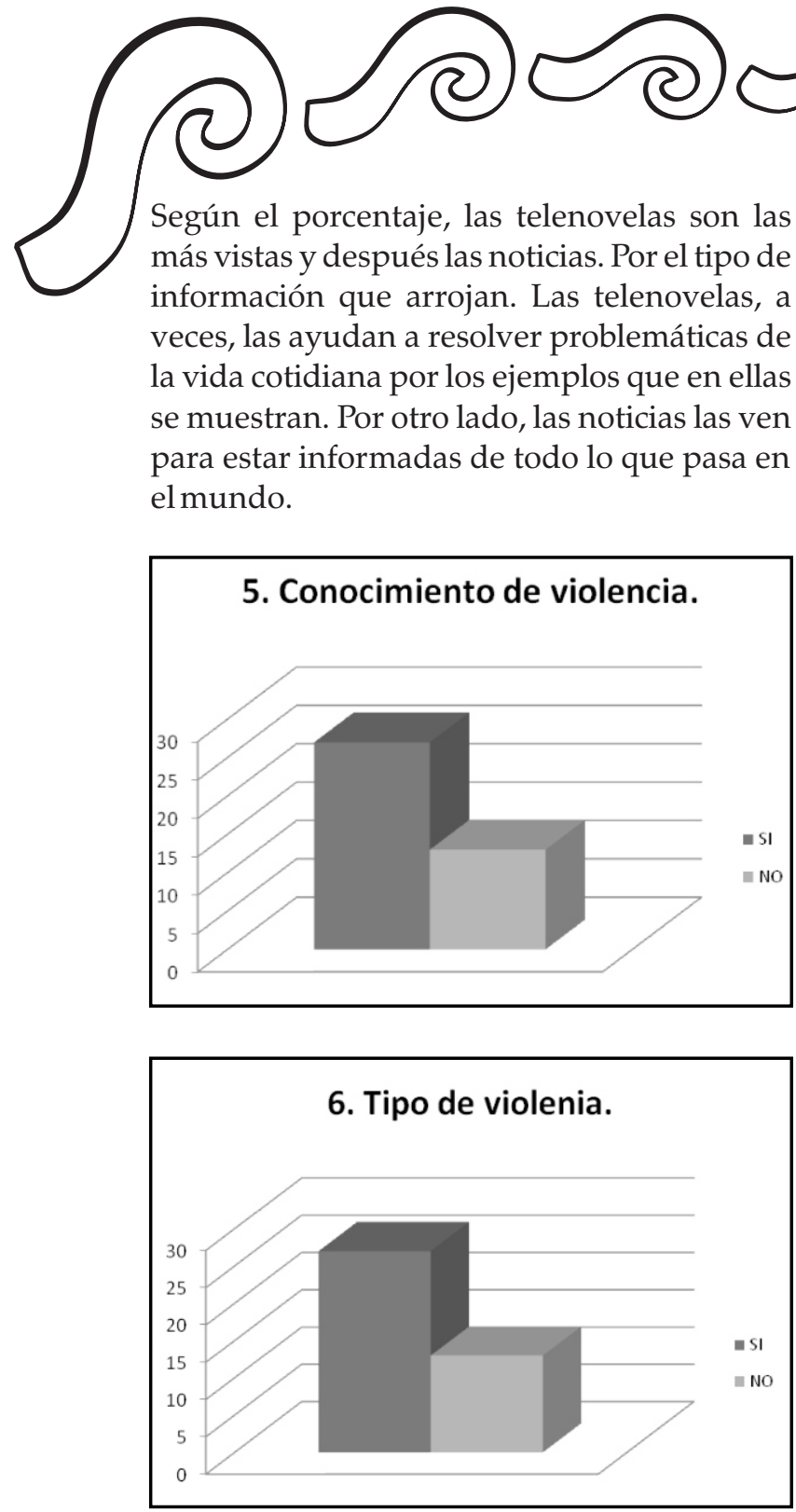

35 mujeres supieron lo que es violencia, la mayoría mencionó que es "Maltrato", otras mencionaron que son "nervios" y otras solo mencionaban los tipos de violencia. Hay una fuerte confusión en la definición y en los tipos de violencia. Algunas señoras nos dijeron que la definición y tipos los sabían por el programa "Oportunidades". Este programa da pláticas en las comunidades con el fin de mantenerlas informadas acerca de temas que no se tratan comúnmente en las comunidades.

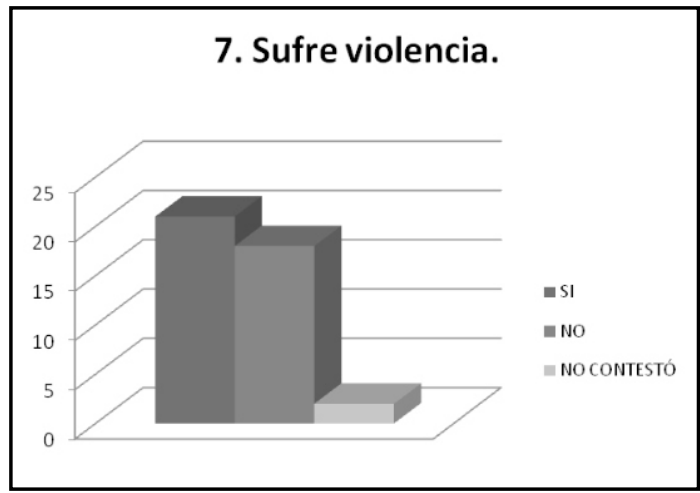

21 mujeres de las comunidades contestaron que sí ha sufrido violencia, pero solo cuando eran pequeñas, no en el presente. Mencionaron que hubo un fuerte cambio en cuanto la educación de los padres, porque antes acostumbraban pegarles cuando no hacían un deber, sin embargo, ahora solo les llaman la atención a los hijos para que estos hagan sus deberes.18 contestaron que nunca han sufrido violencia, de ningún tipo. Pero algunas sí han visto que en sus vecinos se suscitan este tipo de situaciones.

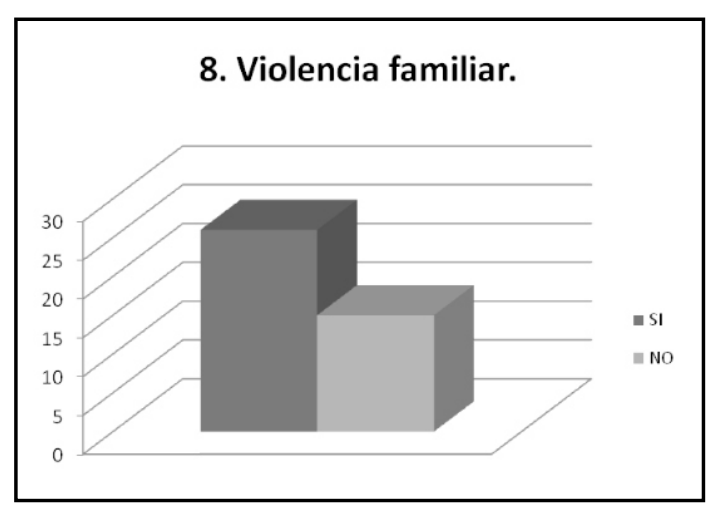

26 mujeres contestaron que creen que sí existe la violencia familiar, pero de manera verbal, al gritarle a sus hijos para que obedezcan. Ninguna mencionó violencia física; dijeron que como los tiempos han cambiado ya no necesitan golpes para que los hijos atiendan sus deberes, con palabras ya hacen lo que deben. Las otras 15 personas dijeron que no existe violencia familiar. 


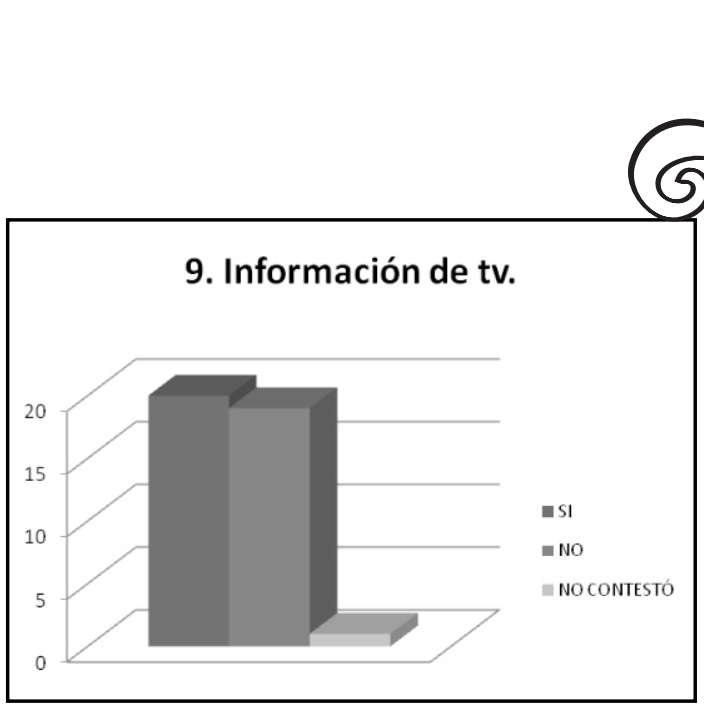

La mayoría de las mujeres contestó que sí se ha informado por medio de la TV, a veces con las noticias y algunas otras con programas como Laura en América. Las personas que contestaron que no era porque no les daba tiempo o van a pláticas del programa Oportunidades, para aprovechar la ayuda que les ofrecen estos tipos de programas.

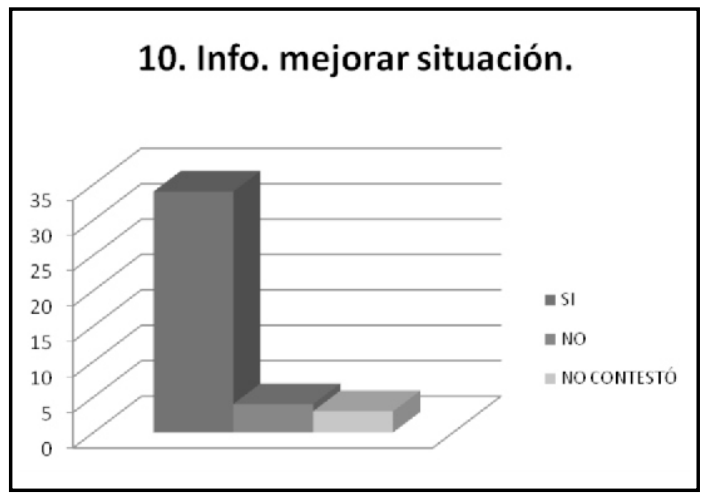

34 mujeres mencionaron que la información que les dan sí les ayuda a mejorar su situación en la comunidad o en su familia, ya sea para defenderse o ayudar a vecinos. Los ejemplos que dan las telenovelas o Laura en América los utilizan como comparación para saber cómo actuar en casos similares. Por otra parte, las pláticas del programa "Oportunidades" las ayudan para evadir este tipo de situaciones, intervenir en situaciones familiares o de la comunidad.
Resultados

El 90\% de la muestra menciona que sí tiene televisión, a la cual el $46 \%$ de ésta le dedica menos de una hora al día y los programas más vistos son las telenovelas con un $41 \%$, seguido de las noticias con un $36 \%$ dichos programas se encuentran dentro de los canales de televisión abierta que resulta más accesible.

El 85\% tenía conocimiento básico acerca de lo que es violencia, definiéndola de diferente manera, como maltrato, golpes, groserías y nervios; el otro $15 \%$ no tenía conocimiento alguno. De este porcentaje el $65 \%$ sabía algunos tipos de violencia.

El 51\% ha sufrido violencia en su vida, de las cuales $11 \%$ mencionaron que la presenciaron cuando eran menores, ya que la violencia se daba de parte de sus padres como autoridad para educarlas. El 5\% no contestó a esta pregunta y el $44 \%$ mencionó que ninguna vez en su vida ha sufrido de violencia de ningún tipo.

$63 \%$ cree que sí existe la violencia familiar actualmente, ya sea de manera física o verbal, ya que escuchan gritos en casa de sus vecinos, en algunos casos las mujeres conocen a otras mujeres que son golpeadas.

El 48\% sí ha utilizado la televisión como medio informativo acerca de la violencia, del cual al $82 \%$ le ha servido esta información. El $46 \%$ menciona que no ha utilizado la televisión para obtener información del tema de violencia.

Una de las teorías con las que podemos relacionar los resultados es "El espiral del silencio" porque algunas de las mujeres encuestadas negaban la situación de violencia o no contestaban nuestras preguntas por el miedo a la difusión de su problema, se mostraban nerviosas e inseguras, esto se comprobó con los instrumentos de observación que ocupamos al realizar las encuestas y platicar con las mujeres. 


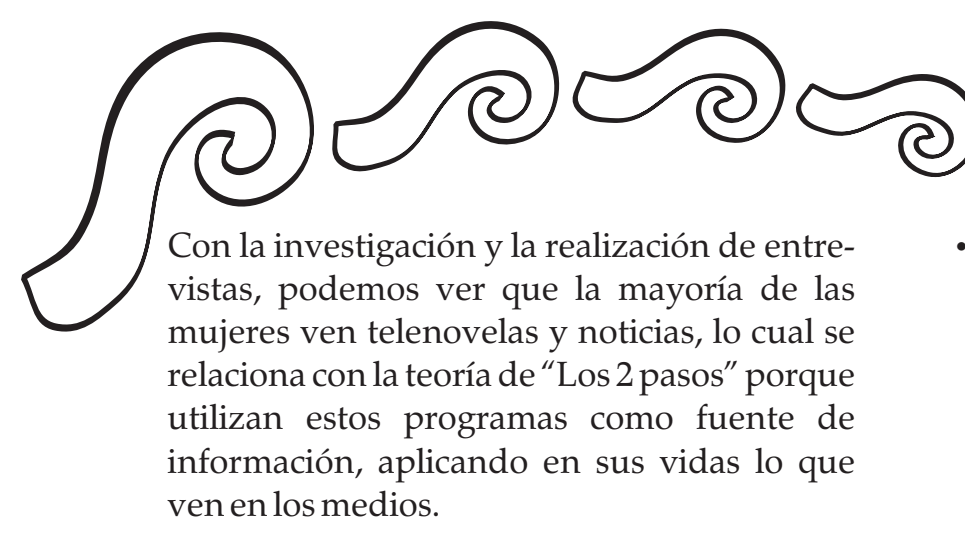

La teoría de La divulgación de las nuevas ideas y prácticas nos ayudó a comprender la forma en que los medios de comunicación influyen en la mejora de la situación de los integrantes de la comunidad, se nota un gran cambio de una generación a otra en la que las mujeres ya están más familiarizadas con la tecnología, tomando en cuenta el contexto en el que se desarrollan, ahora los medios de comunicación como la televisión y la radio son parte de las viviendas.

\section{Conclusiones}

De acuerdo con el objetivo de nuestra investigación, que es identificar los tipos de abuso que se comenten en contra de la mujer y si los medios de comunicación en este caso la televisión era un punto clave de información o desinformación sobre el tema, el resultado de los instrumentos aplicados arrojaron las siguientes conclusiones:

- El51\% de las mujeres ha sufrido violencia, en alguna etapa de su vida, sin embargo la mayoría de ellas aun temen al hablar de estos temas e intentan ocultarlo.

- El 90\% de las mujeres entrevistadas cuentan con televisión en su hogar, aunque menos de la mitad la ve.

- Dentro de los programas más vistos por la mujer se encuentra, Laura que ha funcionado como una fuente de información, ya que se hacen presentes los derechos de las mujeres con distintas temáticas de la vida cotidiana, logrando que la mujer se identifique.
- El $80 \%$ sabe lo que es violencia, sin embargo, no conocen los tipos ni qué hacer en caso de vivir una situación violenta. La poca información que tiene les ha ayudado a mejorar su calidad de vida.

- Dicha información es impartida por el programa Oportunidades que ha creado conciencia sobre sus derechos y las ha hecho autosuficientes, ya que sus esposos trabajan fuera de la comunidad, aunque apoyan con el sustento del hogar, la mujer se ve obligada a llevar el hogar como madre soltera.

\section{Sugerencias}

Para poder generar un gran cambio se debe comenzar con la educación desde la infancia, por ello proponemos talleres o programas informativos dentro de las escuelas en las que el niño reconozca este problema social.

Programas de familia, en las que estén involucrados todos los miembros de ésta, para que la responsabilidad no caiga completamente en la mujer, dichos programas deben ser impartidos a todos los hogares de la comunidad, sin discriminación.

Las autoridades encargadas en las problemáticas de violencia, deben de estar más apegadas a las personas de la comunidad, para que surja un ambiente de confianza.

A la comunidad le serviría el apoyo de La Salle, como autoridad en la que puedan confiar para encaminarlas a las autoridades, ya que la mayoría de las mujeres desconfían de éstas y necesitan una guía.

Los niños deben familiarizarse con la problemática, muchas veces es un tema oculto y por ello pueden surgir rumores, el desconocer una situación crea falsas suposiciones, si los niños conocen de casos y saben que está mal, podrán actuar en contra de ello. 


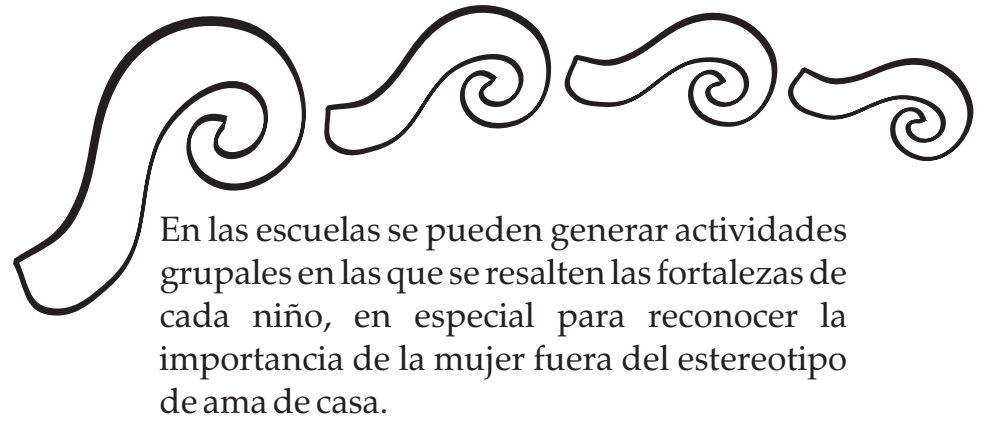

Implementar talleres en los que se den a conocer de una forma aplicada los valores y derechos de cada persona, como niños, mujeres e individuos, aunque las escuelas cuentan con materias de valores, éstas se dan como teoría y lo importante es que las personas las reconozcan y las lleven a su vida.

Planear días específicos para impartir pláticas sobre la violencia a las mamás que viven lejos, para aquellas que no conozcan lo que es violencia y qué hacer en caso de sufrirla o conocer alguien que es violentado.

Apoyo especializado, ya que las mamás de los niños del albergue que son las más cercanas, puedan recibir el apoyo para hacer valer sus derechos, donde las mujeres sepan que cuentan con el apoyo de la escuela para ejercer sus derechos y que las autoridades correspondientes hagan su deber.

Crear un ambiente cariñoso y respetuoso entre niñas y niños, reconocer logros y apoyar en las debilidades.
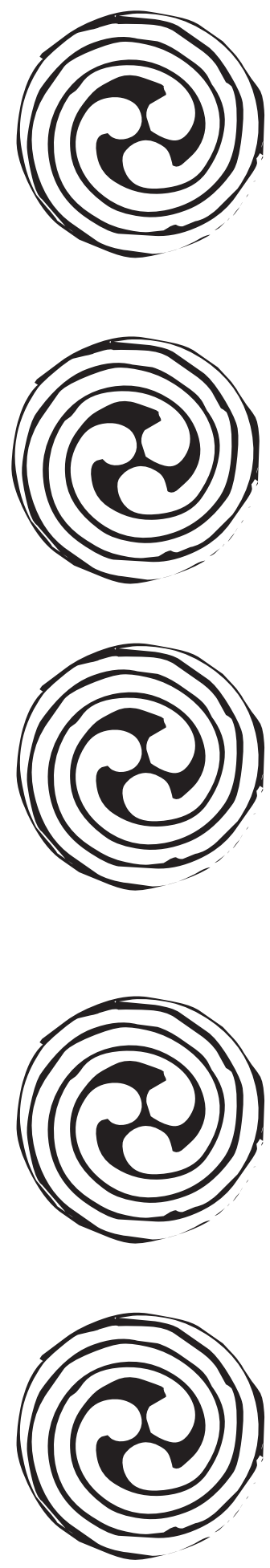


\section{Fuentes de consulta:}

Derecho a las mujeres a una vida libre de violencia en el ámbito familiar. DIF-DIF, 200'

Reglamento y ley de acceso de las mujeres a una vida libre de violencia. DIF-DIF, 2009

http://www.ohchr.org/SP/Issues/Pages/WhatareHumanRigths.aspx)

Portillo Sánchez, Maricela / Esterna Madrid, Javier (1998) Espacios de comunicación, Universidad Iberoamericana. Pp 411-422

Lozano Rendón, José Carlos (1996) Teoría e investigación de la comunicación de masas. Ed Alambra, México Pp. 45-51

Katz, (compilación de Schramm) (1982) "La ciencia de la comunicación" ed. Grijalbo México p. $93-112$

http://www.eluniversal.com.mx/notas/581655.html 24/03/2012

http://www.inmujeres.gob.mx/index.php/ique-es-el-inmujeres/historia 24/03/2012

http://www.inegi.org.mx/est/contenidos/espanol/metodologias/encuestas/hogares/endire h06_marco_concep.pdf 24/03/2012

http://www.inegi.org.mx/sistemas/TabuladosBasicos2/tabulados/envipe/2011/III_caracteris ticas_victimas.PDF 24/03/2012

http://www.inegi.org.mx/Sistemas/temasV2/Default.aspx?s=est\&c=17484 24/03/2012
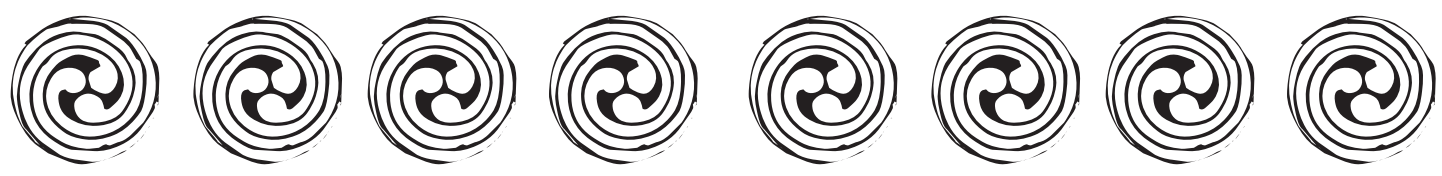
ANEXOS
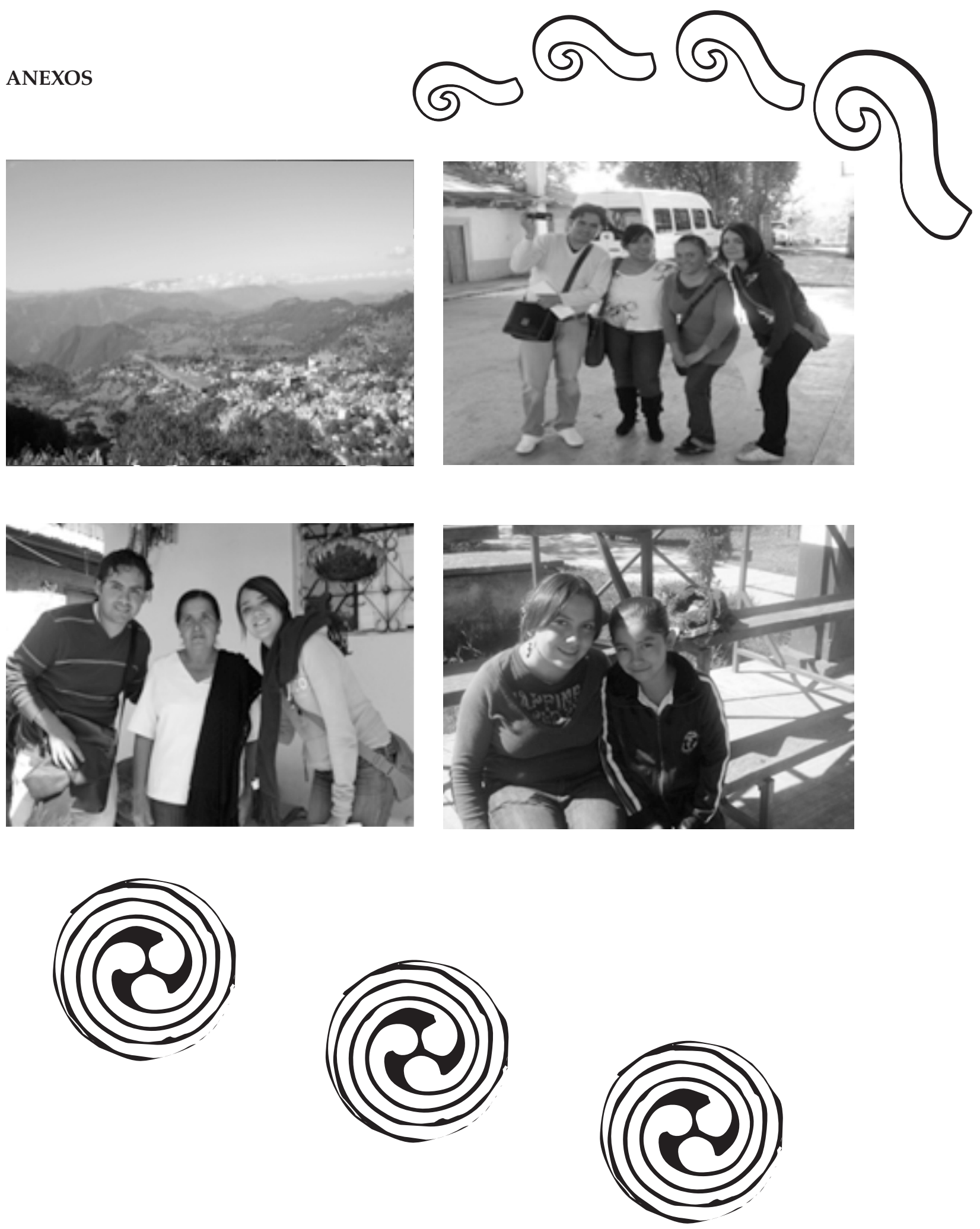


$$
\rho^{\beta}
$$

